\title{
Tiempo de excreción fecal de Escherichia coli productora de toxina Shiga en niños con síndrome urémico hemolítico
}

\author{
Duration of fecal shedding of Shiga toxin-producing \\ Escherichia coli among children with hemolytic uremic syndrome
}

\author{
Dr. Lucas I. Lucarellia, Dra. Laura F. Alconcher ${ }^{a}$, Bq. Verónica Arias ${ }^{a}$ y \\ Bq. Jimena Galavottia
}

\section{RESUMEN}

Introducción. Conocer el tiempo de excreción fecal de Escherichia coliproductora de toxinaShiga (Shiga toxin-producing Escherichia coli; STEC, por sus siglas en inglés) en pacientes con síndrome urémico hemolítico sería útil para controlar la transmisión de la enfermedad.

Objetivos. 1) Analizar las características del tiempo de excreción de STEC. 2) Evaluar la asociación con las variables sexo, edad, necesidad de diálisis, antibióticos y serotipos de STEC.

Población y métodos. Estudio prospectivo, observacional, longitudinal y analítico. Período 2013-2019. Se realizaron coprocultivos al ingresar y cada 5-7 días hasta obtener 2 negativos. Se definió tiempo de excreción desde el inicio de la diarrea hasta el primer negativo. Se confirmó STEC por detección de los genes stx $1, s t x 2$ y rfbO157 por reacción en cadena de la polimerasa. Se calculó la media (IC $95 \%$ ) y percentilos del tiempo de excreción de STEC, y se compararon las variables estudiadas mediante el test de $t$.

Resultados. Se incluyeron 43 pacientes. La media detiempodeexcreción fue 10,2 días (IC95\%:8,9211,59), rango: 3-22 días. El $90 \%$ de los pacientes negativizaron el coprocultivo a los 15 días. No hubo diferencias según sexo $(\mathrm{p}=0,419)$, edad $(\mathrm{p}=0,937)$, necesidad de diálisis $(\mathrm{p}=0,917)$, antibióticos $(p=0,147)$ ni serotipos $(p=0,231)$. Conclusión. El $90 \%$ delos pacientes negativizó el coprocultivo a los 15 días del inicio de la diarrea, y todos, al día 22. No se encontró asociación entre el tiempo de excreción y las variables estudiadas. Palabrasclave: Escherichiacolienterohemorrágica, derrame de bacterias, período de transmisión, heces.

a. Hospital Interzonal Dr. José Penna, Bahía Blanca, Argentina.

Correspondencia: Dr. Lucas Lucarelli: 1_lucarelli@hotmail.com

Financiamiento:

Ninguno.

Conflicto de intereses:

Ninguno que declarar.

http: / / dx.doi.org/10.5546/ aap.2021.39

Texto completo en inglés:

http: / / dx.doi.org/ 10.5546/ aap.2021.eng.39

Cómo citar: Lucarelli LI, Alconcher LF, Arias V, Galavotti J. Tiempo de excreción fecal de Escherichia coli productora de toxina Shiga en niños con síndrome urémico hemolítico. Arch Argent Pediatr 2021;119(1): 39-43.

\section{INTRODUCCIÓN}

El diagnóstico de síndrome urémico hemolítico (SUH) se basa en la tríada diagnóstica anemia hemolítica microangiopática, trombocitopenia y distintos grados de compromiso agudo de la función renal. En la Argentina, su forma de presentación es endémica, con una mediana de casos anuales notificados para el período 2014-2018 de 314 y una mediana de casos para menores de 5 años de 245 . $^{1}$ La mayoría se asocian a diarrea y, en el $65 \%$, se logra confirmar la infección por Escherichia coli productora de toxina Shiga (Shiga toxin-producing Escherichia coli; STEC, por sus siglas en inglés). ${ }^{2}$ La transmisión de la enfermedad se puede producir por la ingesta de un alimento contaminado con STEC, por el contacto con las heces de un animal portador o por el contacto con las heces del enfermo (diarrea y/o SUH) y / o sus contactos cercanos.

Estudios de brotes de diarrea en jardines maternales y de infantes en distintos lugares del mundo han demostrado que la excreción de E. coli $\mathrm{O} 157$ puede ser prolongada e intermitente..$^{3-8}$ Conocer el tiempo de excreción en la materia fecal (MF) de STEC en los pacientes con SUH sería útil para reincorporar en forma segura a estos niños a la sociedad y, así, controlar la transmisión secundaria de la enfermedad.

\section{OBJETIVOS}

1) Analizar las características del tiempo de excreción de STEC en los pacientes con $\mathrm{SUH}$. 
2) Evaluar la asociación entre el tiempo de excreción y las variables sexo, edad, necesidad de diálisis, administración de antibióticos y serotipos de STEC.

\section{MATERIALES Y MÉTODOS}

Estudio prospectivo, observacional, longitudinal y analítico. Se analizaron los resultados de los coprocultivos de los niños con SUH atendidos en el período de enero de 2013-diciembre de 2019 en la Unidad Centinela del Hospital Interzonal General (HIG) Dr. José Penna de Bahía Blanca, Argentina. Las muestras de MF se recolectaron al ingresar al Hospital y cada 5-7 días hasta obtener 2 resultados negativos consecutivos con el fin de detectar casos de excreción intermitente. La confirmación de la infección por STEC se realizó por la detección de los genes st $x_{1}, s t x_{2}$ y $r f b_{\mathrm{O} 157}$ por reacción en cadena de la polimerasa (polymerase chain reaction; PCR, por sus siglas en inglés). ${ }^{9}$

Se excluyeron los pacientes a los que no se les realizó coprocultivo y aquellos con resultado negativo. Se eliminaron los que no cumplieron con el número y/o plazo de entrega de las muestras de MF. Ante la posibilidad de excreción intermitente, se consideró como tiempo de excreción fecal de STEC el transcurrido en días desde el inicio de la diarrea hasta el primero de dos coprocultivos negativos consecutivos separados por $48 \mathrm{~h}$.

Para el cálculo del tamaño muestral necesario para la estimación del tiempo de excreción, se seleccionó una muestra piloto de 12 pacientes, que arrojó un tiempo medio de 19,08 días con un desvío de 13 días. Para una confianza del $95 \%$ y un error absoluto de 4 días (error relativo del $20 \%$ ), se obtuvo un tamaño de muestra necesario

FIgURA 1. Histograma de frecuencias acumuladas de tiempo de excreción de Escherichia coli productora de toxina Shiga en pacientes con sindrome urémico hemolítico

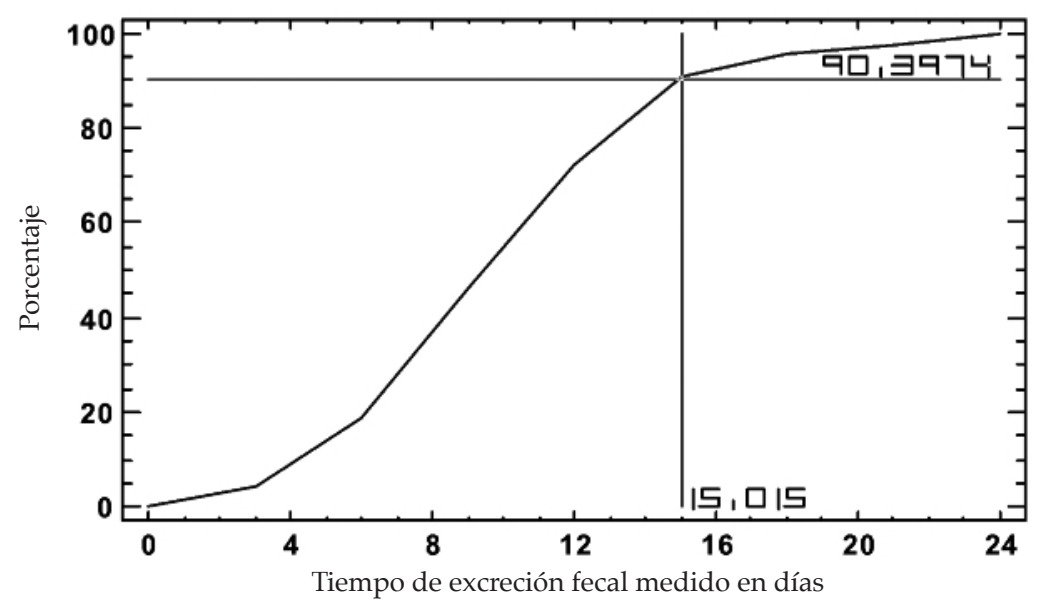

FIGURA 2. Histograma para el tiempo de excreción

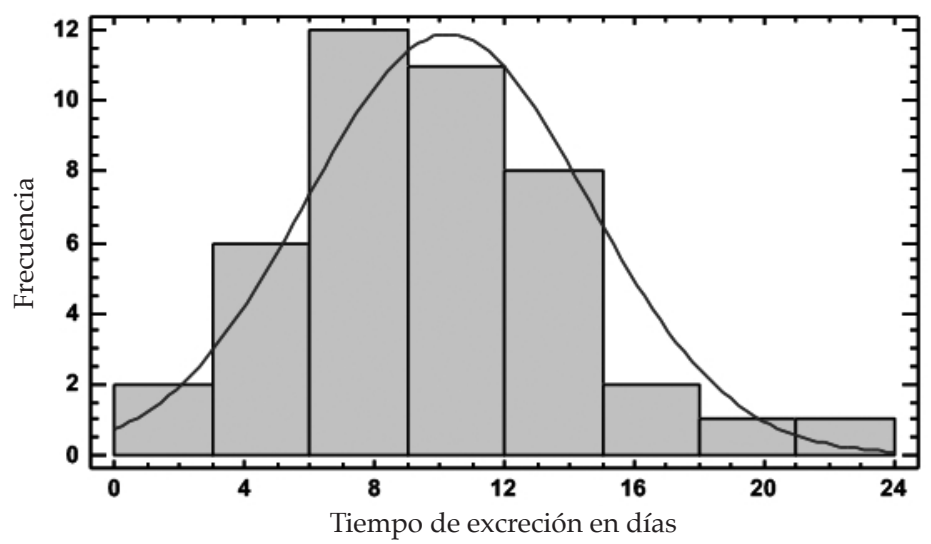

El tiempo de excreción proviene de una distribución normal. Se puede observar el comportamiento de tipo gaussiano. 
de 41 pacientes. Se utilizó el software Epidat 3.1.

Se calcularon la media con su intervalo de confianza (IC) del $95 \%$ y percentilos. Se construyó el histograma de frecuencias acumuladas. Se realizó el test $t$ de diferencias de medias para evaluar la relación del tiempo de excreción con las variables sexo, edad (menor o mayor de 2 años), requerimiento de diálisis (sí/no), administración de antibióticos (sí/no) y serotipos de STEC (O157/no O157). Se consideró significativo un valor de $p<0,05$. Se utilizaron los softwares Statgraphic Centurion XVI y PSSS v22. E1 estudio fue aprobado por los Comités de Ética e Investigación del HIG Dr. J. Penna. Se obtuvieron los consentimientos informados por escrito.

\section{RESULTADOS}

Durante el período 2013-2019, se atendieron 88 pacientes con SUH. Treinta y siete fueron excluidos, 36 por presentar coprocultivo negativo y uno por no estudiarse con coprocultivo. De los 51 pacientes con coprocultivos positivos para STEC, 8 se eliminaron por no cumplir con el número y / o plazo de entrega de las muestras de MF consecutivas.

Se incluyeron 43 pacientes, 22 (el $51 \%$ ) fueron mujeres, y 24 (el $56 \%$ ), menores de 2 años. Veintitrés pacientes (el $53 \%$ ) requirieron diálisis. Cuatro pacientes (el $9 \%$ ) recibieron tratamiento antibiótico antes del ingreso. E. coli $\mathrm{O} 157$ fue aislada en 29 (el $67 \%$ ).

La media de tiempo de excreción fecal fue de 10,2 días (IC $95 \%$ : 8,25-11,39 días). El $90 \%$ de los pacientes tuvieron coprocultivo negativo al día 15, y la totalidad, al día 22 (Figura 1). La variable tiempo de excreción presentó una distribución normal de la curva (Figura 2 y Tabla 1 ).

En solo 2 casos, se detectó excreción fecal intermitente, con un tiempo total de 12 y 21 días,

TABLA 1. Percentilos para el tiempo de excreción fecal de Escherichia coli productora de toxina Shiga en pacientes con sindrome urémico hemolítico $(n=43)$

\begin{tabular}{lc}
\hline Percentilo & Días de excreción \\
\hline $1 \%$ & 3 \\
$5 \%$ & 4 \\
$10 \%$ & 5 \\
$25 \%$ & 7 \\
$50 \%$ & 10 \\
$75 \%$ & 13 \\
$90 \%$ & 15 \\
$95 \%$ & 17 \\
$99 \%$ & 22 \\
\hline
\end{tabular}

respectivamente. No se encontró asociación estadísticamente significativa entre el tiempo de excreción fecal de STEC y la edad, el sexo, el requerimiento de diálisis, el uso de antibióticos ni el serotipo detectado (Tabla 2).

\section{DISCUSIÓN}

Como ha sido comunicado en diversos estudios epidemiológicos, la transmisión de STEC persona-persona juega un papel importante en el comportamiento endémico del SUH en la Argentina. Dicha transmisión está ligada, en especial, a los jardines de infantes, maternales y a los contactos familiares cercanos. . $^{510,11}$ Recientemente, se observó que un tercio de los pacientes con $S U H$ tenían contactos familiares STEC positivos, la mayoría asintomáticos, pero con potencial riesgo de transmisión. ${ }^{10} \mathrm{La}$ transmisión interpersonal es muy fácil, debido a que la dosis infectiva es muy baja (100-500 organismos). ${ }^{11}$

Belongia y col. (1993) no evidenciaron transmisión persona-persona de STEC en jardines maternales cuando los niños con diarrea eran reincorporados a la institución luego de 2 coprocultivos negativos. ${ }^{7}$ Este mismo criterio es el utilizado en nuestro país para la reincorporación a jardines de infantes y maternales en pacientes con $\mathrm{SUH}$, pero la detección de $S T E C$ en la MF por PCR es un estudio costoso, complejo y no accesible en todos los hospitales. Por este motivo, conocer el tiempo de excreción fecal de STEC podría ser una herramienta útil para la prevención de nuevos

TABLA 2. Relación entre el tiempo de excreción de Escherichia coli productora de toxina Shiga en niños con síndrome urémico hemolítico con variables clínicas, demográficas y microbiológicas seleccionadas $(n=43)$

\begin{tabular}{lcccc}
\hline Factor & Categoría & N (días) & Media & Valor de $\boldsymbol{p}$ \\
\hline Sexo & Varón & 21 & 10,81 & 0,419 \\
& Mujer & 22 & 9,73 & \\
Edad (años) & $<2$ & 24 & 10,21 & 0,937 \\
\multirow{2}{*}{ Serotipos de STEC } & No O157 & 14 & 10,36 & 0,917 \\
\multirow{2}{*}{ Antibióticos } & O157 & 29 & 10,21 & \\
& Sí & 4 & 7,25 & 0,147 \\
Requerimiento & No & 39 & 10,56 & \\
de diálisis & & & & \\
& Sí & 23 & 11,00 & 0,231 \\
& No & 20 & 9,40 & \\
\hline
\end{tabular}

STEC: Escherichia coli productora de toxina Shiga. 
casos. En nuestro trabajo, se demostró que el $90 \%$ de los pacientes con SUH negativizaron el coprocultivo a los 15 días de haberse iniciado la diarrea, y la totalidad, a los 22 días.

La mayor información sobre el tiempo de excreción fecal de STEC proviene de pacientes con diarrea con serotipo O157. Shash y col. (1996), al estudiar un brote en un jardín maternal, comunicaron un tiempo medio de excreción de E. coli O157 de 29 días (rango: 11-57 días). ${ }^{4}$ Miliwebsky y col. (2007) estudiaron 4 eventos de diarrea ocurridos en jardines maternales de la Argentina que afectaron a niños, personal del jardín y familiares. En tres de ellos, el serotipo aislado fue no O157. La excreción fue prolongada y similar entre los serotipos O157 y no O157 (con un máximo de 37 y 31 días para el serotipo O26:H11 y de 19 días para O145:NM) ${ }^{5}$

Pocos datos han sido comunicados en pacientes con SUH. Karch y col. (1995) estudiaron a 53 pacientes con diarrea por STEC y a 25 pacientes con $S U H$, y encontraron que la media de tiempo de excreción fecal fue significativamente mayor en los pacientes con SUH (13 vs. 21 días, respectivamente, $p<0,001){ }^{8}$ A la inversa, en el brote de diarrea y SUH causado por E. coli enteroagregativa O104:H4 en 2011 en Alemania, el tiempo de excreción fecal de STEC tuvo una mediana de 13-14 días en los pacientes con SUH vs. 33-34 días en los pacientes con diarrea. ${ }^{12}$ Excreciones más prolongadas podrían deberse a una dosis infectiva mayor de $10^{6}$ unidades formadoras de colonias (UFC) $/ \mathrm{g} .{ }^{8} \mathrm{En}$ 2015, Matussek comunicó un tiempo de excreción fecal promedio de STEC de 23 días en 7 pacientes con SUH y de 20 días en pacientes con diarrea. ${ }^{13}$

El tratamiento con antibióticos de un paciente con diarrea por STEC puede incrementar el riesgo de desarrollar SUH y, por este motivo, no se recomienda. ${ }^{14}$ Sin embargo, en el brote de Alemania, el grupo de pacientes con SUH grave que se trató con eculizumab y recibió azitromicina, como profilaxis para disminuir el riesgo de meningitis, mostró un tiempo de excreción de STEC significativamente más corto. Solo un paciente de 22 tratados con azitromicina (el 4,5\%) tuvo una excreción mayor de 28 días vs. 35 de 43 (el $81 \%$ ) de los que no recibieron antibióticos. ${ }^{15}$ Solo 4 pacientes en nuestra serie habían recibido antibióticos antes del ingreso. Si bien el tiempo de excreción fue más corto en estos pacientes, no alcanzó significancia estadística. No existen, a la fecha, datos concluyentes sobre la utilidad de los antibióticos para acortar el tiempo de excreción de STEC en los pacientes con SUH.

Aunque se ha comunicado una excreción de STEC más prolongada en pacientes de menor edad, tanto en brotes de diarrea como en pacientes con $\mathrm{SUH}$, no se encontró, en nuestro estudio, dicha asociación estadística. ${ }^{3,12} \mathrm{Si}$ bien existen datos ya publicados sobre el tiempo de excreción de STEC, la mayoría son trabajos de pacientes con diarrea y algunos casos aislados de $\mathrm{SUH}$, excepto en el grupo de Alemania, que estudió otra categoría de E. coli.

E1 $90 \%$ de los pacientes negativizó el coprocultivo a los 15 días del inicio de la diarrea. Por disposición del Ministerio de Salud, los pacientes con $\mathrm{SUH}$ no deben ser reintegrados a jardines infantiles y/o maternales hasta no tener, al menos, 2 coprocultivos consecutivos negativos. En caso de no contar con esta posibilidad, y considerando la potencial gravedad de una infección por STEC, con la probabilidad de desarrollar $\mathrm{SUH}$, parece prudente adoptar 22 días como límite de seguridad para retomar las actividades sociales. Conocer este dato permite determinar cuándo un paciente puede ser reintegrado a la sociedad con bajo riesgo de transmisibilidad. Se cree que esta información puede ser especialmente útil en la Argentina para la prevención de la aparición de nuevos casos, debido al comportamiento endémico de la enfermedad y al importante papel de la transmisión interpersonal.

\section{CONCLUSIÓN}

E1 $90 \%$ de los pacientes negativizó el coprocultivo a los 15 días del inicio de la diarrea, y todos, al día 22. No se encontró asociación estadísticamente significativa entre el tiempo de excreción fecal de STEC y la edad, el sexo, el requerimiento de diálisis, el uso de antibióticos ni el serotipo detectado. Estos datos podrían ser útiles para prevenir la aparición de casos secundarios de infección de STEC en aquellos sitios donde no puedan realizar la búsqueda de la negativización por $P C R$.

\section{Agradecimientos}

A las Doctoras en Economía María Eugenia Elorza y Fernanda Villarreal por el análisis estadístico realizado, Universidad Nacional del Sur.

\section{REFERENCIAS}

1. Argentina. Ministerio de Salud. Vigilancia de Síndrome Urémico Hemolítico. Boletín Integrado de Vigilancia. 2019;477(SE 50):47. [Acceso: 11 dejunio de 2020]. Disponible 
en: https: / / www.argentina.gob.ar/sites/default/ files/201912-biv_477.pdf.

2. Rivas M. Epidemiología del Síndrome Urémico Hemolítico en Argentina. Situación actual e innovaciones diagnósticas. 2016. [Acceso: 11 dejunio de2020]. Disponible en: http: / / www.sap.org.ar/uploads/archivos / / files_dra-rivas-epidemiologia-del-sindrome-uremicohemolitico-en-argentina-situacion-actual-e-innovacionesdiagnosticas_1494446234.pdf.

3. Swerdlow DL, Griffin PM. Duration of faecal shedding of Escherichia coli O157:H7 among children in day-care centres. Lancet. 1997; 349(9054):745-6.

4. Shash S, Hoffman R, Shillam P, Wilson B. Prolonged fecal shedding of Escherichia coli O157:H7 during an outbreak at day care center. Clin Infect Dis. 1996; 23(4):835-6.

5. Miliwebsky E, Deza N, Chinen I, Martínez Espinosa E, et al. Prolonged fecal shedding of Shiga toxin-producing Escherichia coli among children attending day-care centers in Argentina. Rev Argent Microbiol. 2007; 39(2):90-2.

6. Raffaelli RM, Paladini M, Hanson H, Kornstein L, et al. Child care-associated outbreak of Escherichia coli O157:H7 and hemolytic uremic syndrome. Pediatr Infect Dis J. 2007; 26(10):951-3.

7. Belongia EA, Osterholm MT, Soler JT, Ammend DA, et al. Transmission of Escherichia coli 0157:H7 Infection in Minnesota Child Day-care Facilities. JAMA. 1993; 269(7):883-8.

8. Karch H, Rüssman H, Schmidt H, Schwarzkopf A, et al. Long-term shedding and clonal turnover of enterohemorrhagic Escherichia coli O157:H7 in diarrheal diseases. J Clin Microbiol. 1995; 33(6):1602-65.
9. Servicio deFisiopatogenia, Departamento de Bacteriología. Manual de Procedimientos. Diagnóstico y Caracterización de Escherichia coli productor de toxina Shiga O157 y noO157 a partir de especímenes clínicos. Buenos Aires: INEIANLIS “Carlos G. Malbrán"; 2011.

10. Alconcher LF, Rivas M, Lucarelli LI, Galavotti J, et al. Shiga toxin-producing Escherichia Coli in household members of children with hemolytic uremic syndrome. Eur J Clin Microbiol Infect Dis. 2020; 39(3):427-32.

11. Zotta CM, Chinen I, Lavayén S, Cepeda M, et al. Portación de Escherichia coli en convivientes de casos de síndrome urémico hemolítico. Salud(i)Ciencia. 2015; 21(2):136-41.

12. Vonberg R, Höhle M, Aepfelbacher M, Bange FC, et al. Duration of fecal shedding of Shiga toxin-producing Escherichia coli O104:H4 in patients infected during the 2011 outbreak in Germany: a multicenter study. Clin Infect Dis. 2013; 56(8):1132-40.

13. Matussek A, Einemo I, Jogenfors A, Löfdahl S, et al. Shiga toxin-producing Escherichia coli in diarrheal stool of Swedish children: evaluation of polymerase chain reaction screening and duration of shiga toxin shedding. J Pediatric Infect Dis Soc. 2016; 5(2):147-51.

14. Wong CS, Jelacic S, Habeeb RL, Watkins SL, et al. The risk of the hemolytic-uremicsyndrome after antibiotic treatment of Escherichia coli O157:H7 infections. N Engl J Med. 2000; 342(26):1930-6

15. Nitschke M, Sayk F, Hartel C, Härtel C, et al. Association between azithromycin therapy and duration of bacterial shedding among patients with Shiga toxin-producing enteroaggregative Escherichia coli O104:H4. JAMA. 2012; 307(10):1046-52.

\section{Archivos hace 75 años}

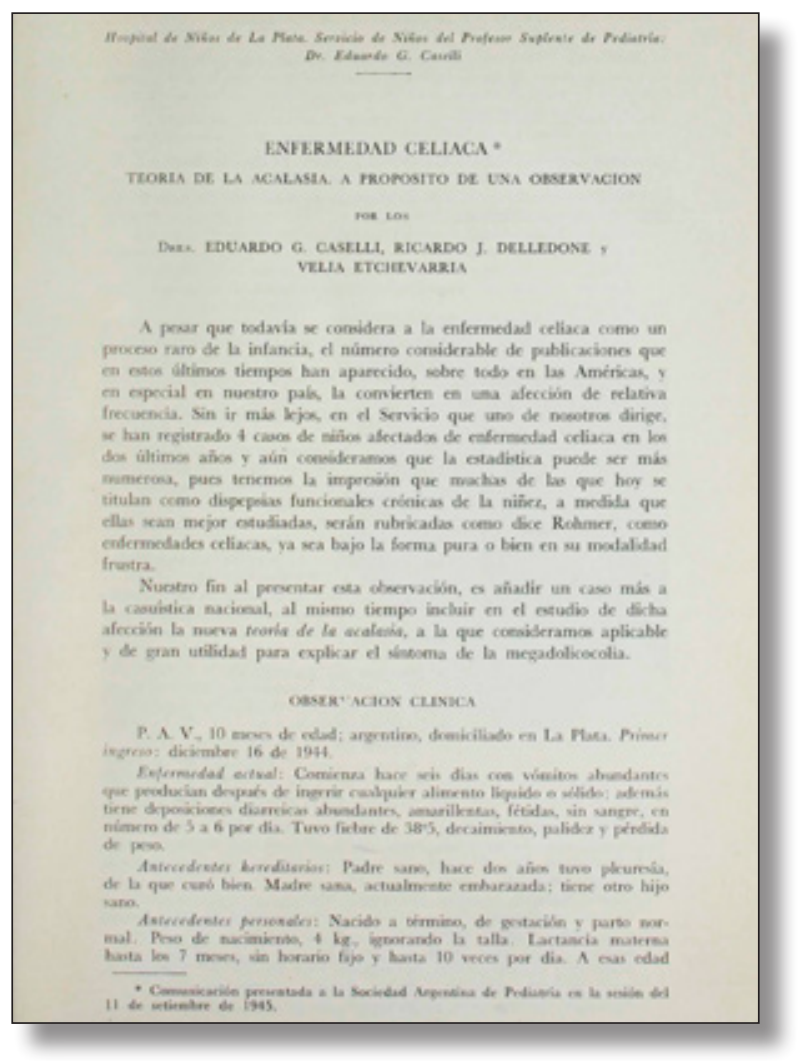

El texto completo se encuentra disponible en la versión electrónica de este número. 